\title{
Obesity: A Risk Marker or an Independent Risk Factor for Coronary Artery Disease?
}

\author{
Tufi Dippe Jr. ${ }^{(\mathbb{C}}$ and Rodrigo Julio Cerci ${ }^{(\mathbb{C}}$ \\ Quanta Diagnóstico por Imagem, Curitiba, PR - Brazil
}

We congratulate Pereira et al. for their study investigating the role of obesity as a risk factor for coronary artery disease (CAD) using coronary computed tomography angiography. The study included 1,383 patients, none with a history of CAD. ${ }^{1}$

Obesity is a multifactorial chronic disease, characterized by accumulation of subcutaneous and visceral fat, that predisposes individuals to metabolic disorders. Several mechanisms are involved in the association between obesity and atherosclerosis, including lipid abnormalities, insulin resistance, chronic inflammation, endothelial dysfunction, reduction of fibrinolysis and hypercoagulability. In addition, the production of proinflammatory cytokines, such as IL-6, TNF-alpha, MCP-1 and leptin contributes to chronic subclinical inflammation. More recent data have indicated that impaired autophagy and altered gut microbiome homeostasis are contributing factors for the development of atherosclerosis in obese individuals. ${ }^{2}$

In the study by Pereira et al., ${ }^{1}$ the authors demonstrated, using coronary computed tomography angiography, that the prevalence of obstructive CAD was similar between obese and non-obese individuals, with a similar prevalence of cardiovascular risk factors. However, mean calcium score was significantly higher in obese than non-obese subjects. Another important finding was that, after multivariate analysis, the clinical variables age, male sex and diabetes mellitus were associated with the presence of obstructive CAD. In this study, obesity was not correlated with CAD.

In a recent publication by our group, we studied a large population of obese individuals without known CAD

\section{Keywords}

Obesity; Risk Factors; Atherosclerosis; Coronary Artery Disease/physiopathology; Metabolic Syndrome; Angiography, Coronary. using myocardial perfusion scintigraphy. We found that age, diabetes mellitus, typical chest pain, poor exercise capacity during stress test, need for pharmacological protocol and low left ventricular ejection fraction were associated with abnormal perfusion. In our study, we did not detect a higher percentage of perfusional abnormalities with increasing degree of obesity. ${ }^{3}$

It is unquestionable the association between obesity, atherosclerosis, and increased total and cardiovascular mortality; however, medical research on the theme has led to controversial results. The first controversy is whether obesity is an independent risk factor for CAD or merely a risk marker, as it is associated with many pathophysiological mechanisms involved in the development of atherosclerosis. Prospective, cohort observational studies, such as the Framingham Study, the Nurses' Health Study and the Health Professionals Follow-Up Study have demonstrated an almost linear relationship between a body mass index (BMI) $\geq 25 \mathrm{~kg} / \mathrm{m}^{2}$ and risk of CAD. Besides, the Framingham cohort study pointed towards obesity as an independent risk factor for atherosclerosis, although these findings were not confirmed by the Seven Countries Study. ${ }^{4}$ Another evidence for obesity as an independent risk factor for atherosclerosis emerged from the Pathobiological Determinants of Atherosclerosis in Youth (PDAY) study. In this study, the researchers, through pathological examination, analyzed coronary arteries and aorta of young people aged 15 to 30, who had accidental deaths or committed suicide.The authors found that atherosclerosis starts early in life, and that obesity is associated with higher prevalence of coronary atherosclerotic lesions, such as fatty streaks, atheroma, and complex coronary lesions, especially in men. ${ }^{4}$

Another controversial point in the association between obesity and atherosclerosis is the "metabolically healthy obesity" phenotype, or MHO. The controversy starts with the MHO's definition, since the diagnostic criteria varied among the studies, resulting in variations in the

Mailing address: Tufi Dippe Jr.

Rua Rocha Pombo, 920 apto. 501. Postal Code: 80530-290, Juvevê, Curitiba, PR - Brazil.

E-mail: tufidippejr@gmail.com 
prevalence of obesity, ranging from $8 \%$ to $40 \% .{ }^{5}$ Ortega et al. ${ }^{5}$ proposed a universal classification of $\mathrm{MHO}$, which should be diagnosed by the absence of all diagnostic elements of the metabolic syndrome (MS) proposed by the International Diabetes Federation, except for abdominal circumference, as follows - blood pressure $\geq 130 / 85 \mathrm{mmHg}$ (or antihypertensive drug treatment), HDL-cholesterol $<40 \mathrm{mg} / \mathrm{dL}$ (or drug treatment for this condition), triglycerides $>150 \mathrm{mg} / \mathrm{dl}$ ( $\mathrm{dL}$ (or drug treatment for this) and fasting glucose $\geq 100 \mathrm{mg} / \mathrm{dl}$ (or treatment for this condition). ${ }^{5}$

The most correct study in terms of methodology, that evaluated the relationship between $\mathrm{MHO}$ and atherosclerosis by coronary computed tomography angiography, was the study by Chang et al. ${ }^{6}$ The authors concluded that $\mathrm{MHO}$ individuals had a higher prevalence of coronary calcification than normal weight individuals. However, such association was no longer statistically significant after adjustment of the values for the metabolic risk factors. This fact suggests that, in MHO subjects, this association occurred at higher (but still normal) levels. One limitation of this study is that it was conducted with a relatively young (mean age of 39.8 years), homogenous population of Asian patients, making the extrapolation of results to other populations difficult. ${ }^{6}$

Previous studies that did not show an association between $\mathrm{MHO}, \mathrm{CAD}$ and cardiovascular risk had methodological limitations, such as the presence of one of the MS diagnostic criteria (in addition of abdominal circumference); the authors did not detect new-onset metabolic changes in $\mathrm{MHO}$ individuals after inclusion in the study; and control groups were composed of overweight subjects rather than metabolically healthy or normal weight individuals. ${ }^{6}$

Another important consideration is that $\mathrm{MHO}$ is not a stable condition, and hence should not be considered as a reliable clinical parameter for predicting cardiovascular risk. Mongraw-Chaffin et al. ${ }^{7}$ followed 6,809 participants of the MESA (Multi-Ethnic Study of Atherosclerosis) for approximately 12 years. The authors concluded that although MHO individuals had a low risk of developing cardiovascular diseases in the beginning of the study, at least $50 \%$ of them developed the metabolically unhealthy obesity (MUO). The presence and duration of MUO was strongly associated with the development of cardiovascular disease and increased mortality. ${ }^{7}$ A large cohort study, evaluating individuals with $\mathrm{MHO}$, concluded that weight lost, particularly greater than $5 \%$ of body weight was associated with lower incidence of atherosclerosis, assessed using carotid artery ultrasonography. ${ }^{8}$

The prevalence of obesity has increased in the last three decades, reaching epidemic proportions in the world. In Brazil, recent data from VIGITEL, a surveillance system for risk factors for chronic diseases by telephone survey, showed that $56 \%$ of Brazilians were overweight and $20 \%$ were obese in $2018 .{ }^{9}$

Although it has not been precisely defined whether obesity is an independent risk factor or simply a risk marker, its association with atherosclerosis, and total and cardiovascular mortality is unquestionable. MHO should not be considered a benign or stable condition, since it frequently progresses to MS, which has been proven to increase cardiovascular risk. In conclusion, obesity must be prevented and treated since childhood.

\section{References}

1. Pereira LLS, Moraes GM, Castro Carneiro AC, Moreira V, Bello JH, Prazeres CE, et al. Relationship between Obesity and Coronary Artery Disease Defined by Coronary Computed Tomography Angiography. Int J Cardiovasc Sci. 2020;33(1):57-64.

2. Lovren F, Teoh H, Subodh V. Obesity and Atherosclerosis: Mechanistic Insights. Can J Cardiol. 2015; 31(2):177-83.

3. Dippe Jr. T, Cunha CLP, Cerci RJ, Stier Jr AL, Vitola JV, et al. Study of Myocardial Perfusion in Obese Individuals without Known Ischemic Heart Disease. Arq Bras Cardiol. 2019; 11S, et al.2(2)a:121-8.

4. Mandviwala T, Khalid U, Deswal A. Obesity and Cardiovascular Disease: a Risk Factor or a Risk Marker.Curr Atheroscler Rep. 2016;18(5):21.

5. Ortega FB, Lavie CJ, Blair S N. Obesity and Cardiovascular Disease. Circulation Research. 2016;118(11):1752-70.

6. Chang Y, Kim BK, Yun KE, Cho J, Zhang Y, Rampal S, et al. Metabolically healthy obesity and coronary artery calcification. J Am Coll Cardiol. 2014; 63(24):2679-86

7. Mongraw-Chaffin M, Foster MC, Anderson CAM, Burke GL, Haq N Kalyani R. et al. Metabolically Healthy Obesity, Transition to Metabolic Syndrome and Cardiocascular Risk. J Am Coll Cardiol. 2018;71(17):1857-65.

8. Sinn DH, Kang D, Cho SJ,Chang Y, Ryu S, Song YB. et al. Weight Change and Development of Subclinical Carotid Atherosclerosis Among Metabolically Healthy Adults: A Cohort Study. J Clin Endocrinol Metab. 2019;104(11):145-63.

9. Brasil.Ministério da Saúde.Vigitel Brasil 2018. Vigilância de fatores de risco e proteção para doenças crônicas por inquérito telefônico. .[Internet] [Citado em 2019 jul 30]. Disponível em: portalarquivos2.saude.gov.br/ images/pdf/2019/julho/25/vigitel-brasil-2018.pdf 